\title{
Uterine perforation and its dosimetric implications in cervical cancer high-dose-rate brachytherapy
}

\author{
Yasir A. Bahadur, MD!, Maha M. Eltaher, MDI,2, Ashraf H. Hassouna, MD2,3, Mohammad A. Attar, MD', \\ Camelia Constantinescu, PhD ${ }^{4}$ \\ 'Department of Radiology, King Abdulaziz University Hospital, Jeddah, Saudi Arabia, ${ }^{2}$ Department of Radiation Oncology, National \\ Cancer Institute, Cairo University, Egypt, ${ }^{3}$ Department of Oncology, King Faisal Specialist Hospital \& Research Center, Jeddah, Saudi Arabia, \\ ${ }^{4}$ Department of Bio-Medical Physics, King Faisal Specialist Hospital \& Research Center, Jeddah, Saudi Arabia
}

\begin{abstract}
Purpose: To retrospectively assess the incidence of sub-serosal and uterine perforation of intra-uterine tandem in intracavitary high-dose-rate (HDR) brachytherapy for cervical cancer, and to evaluate its dosimetric implications on computed tomography (CT)-based treatment planning.

Material and methods: Computed tomography images and brachytherapy plans of cervical cancer patients treated from February 2006 to December 2012 were reviewed for sub-optimal implants (sub-serosal and uterine perforation), and their correlation with cancer FIGO stage and patients' age. For each patient, the plans showing sub-optimal insertion of intra-uterine tandem were analyzed and compared to plans with adequate insertion. The difference in dose coverage of clinical-target-volume (CTV) and variation of the dose delivered to organs-at-risk (OARs) rectum and bladder were evaluated.

Results: A total of 231 brachytherapy plans for 82 patients were reviewed. We identified $12(14.6 \%)$ patients and $14(6 \%)$ applications with uterine perforation, and 12 (14.6\%) patients and $20(8.6 \%)$ applications with sub-serosal insertion of tandem. Data analysis showed that advanced stage correlates with higher incidence of sub-optimal implants $(p=0.005)$ but not the age $(p=0.18)$. Dose-volume-histograms (DVHs) analysis showed large variations for CTV dose coverage: $\mathrm{D}_{90}$ significantly decreased with average of $-115.7 \% \pm 134.9 \%$ for uterine perforation and $-65.2 \% \pm 82.8 \%$ for sub-serosal insertion $(p=0.025)$. The rectum and bladder dose assessed by $\mathrm{D}_{2 \mathrm{cc}}$ increased up to $70.3 \%$ and $43.8 \%$, respectively, when sub-optimal insertion of uterine tandem occurred.

Conclusions: We report a low incidence of uterine perforation and sub-serosal insertion of uterine tandem in intracavitary HDR brachytherapy for cervical cancer. However, the effects on treatment plan dosimetry can be considerably detrimental. Therefore, we recommend image-guided insertion, at least for the challenging cases.
\end{abstract}

Key words: cervix brachytherapy, cervical cancer, uterine perforation.

\section{Purpose}

Locally advanced cervical cancer is optimally treated by concurrent chemo-radiotherapy [1-3]. Radiotherapy includes external beam radiotherapy (EBRT), followed by a brachytherapy boost. Several studies have shown that the outcome of patients treated for bulky and advanced stage disease is directly correlated with the combined dose of radiation delivered by EBRT and brachytherapy [4-6]. Intracavitary brachytherapy involves insertion of a uterine tandem into the cervix and uterus. Sub-optimal insertion of uterine tandem can perforate the uterus and can result in increased dose to nearby organs-at-risk (OARs). A perforation rate of $8 \%$ has been reported in applicators insertions, even if the operator is confident of placement $[7,8]$.
Uterine perforation is a potential complication of uterine tandem insertion, as a result of the tandem being inserted in a false passage outside the uterine canal. If perforation remains undetected and radiation delivered, this can significantly affect the treatment outcome [9-11].

The purpose of this study was to retrospectively assess the incidence of sub-serosal and uterine perforation in intracavitary high-dose-rate (HDR) brachytherapy for cervical cancer, and to evaluate its effects on computed tomography (CT)-based treatment planning dosimetry.

\section{Material and methods}

Cervical cancer patients treated with HDR brachytherapy from February 2006 to December 2012 at the Radio- 
therapy Unit of King Abdulaziz University Hospital, Jeddah, Saudi Arabia, were retrospectively identified. The study was reviewed and approved by the institutional Research Ethics Committee.

All brachytherapy applications were performed under general anesthesia, without radiological guidance for uterine tandem insertion, by a radiation oncologist. Computed tomography images were then acquired using a Siemens Somatom Emotion CT-scanner (Siemens Medical Systems, Erlangen, Germany), with $2 \mathrm{~mm}$ slice intervals from the iliac crest to the distal end of the applicator.

The clinical-target-volume (CTV) and OARs volumes were defined on axial CT images for each brachytherapy fraction at the time of treatment planning, and retrospectively reviewed by one radiation oncologist, for the purpose of this study. The gross-target-volume (GTV) was delineated based on the CT information at the time of the brachytherapy and clinical findings. Isotropic margin (usually $1 \mathrm{~cm}$ ) was added to generate the CTV. Primarily, the cervix and the lower uterine segment were included. If there was an involvement of the fornices or proximal vagina, these were also encompassed. Organ-at-risk volumes were the rectum and bladder. The outer rectal wall was contoured from the recto-sigmoid junction till $1 \mathrm{~cm}$ above the anal verge, and the outer bladder wall was contoured till the urethra.

Demographic and clinical data such as: patients' age, International Federation of Gynecology and Obstetrics (FIGO) stage, initial tumor volume and residual tumor volume after EBRT were collected.

Computed tomography-based treatment plans were reviewed for uterine perforation or near-perforation (sub-serosal insertion of tandem, i.e. within $5 \mathrm{~mm}$ from the uterine serosa), as presented in Figure 1. For each patient, the plans showing sub-optimal insertion of intra-uterine tandem were analyzed and compared to plans with optimal insertion for the same patient. The difference in dose coverage of CTV was evaluated by the doses delivered to $90 \%\left(D_{90}\right)$ and $100 \%\left(D_{100}\right)$ of CTV. The variation of the dose received by $1 \mathrm{cc}\left(\mathrm{D}_{1 \mathrm{cc}}\right)$ and $2 \mathrm{cc}\left(\mathrm{D}_{2 \mathrm{cc}}\right)$ of OARs was also determined and analyzed to assess any possible correlation with the position of the uterine tandem relative to the uterus. All CT-based brachytherapy plans were generated using Varian Brachyvision planning system, versions 8.0 and 10.0, for Varian HDR VariSource 200 and iX (Varian Medical Systems Inc., Palo Alto, CA, USA).
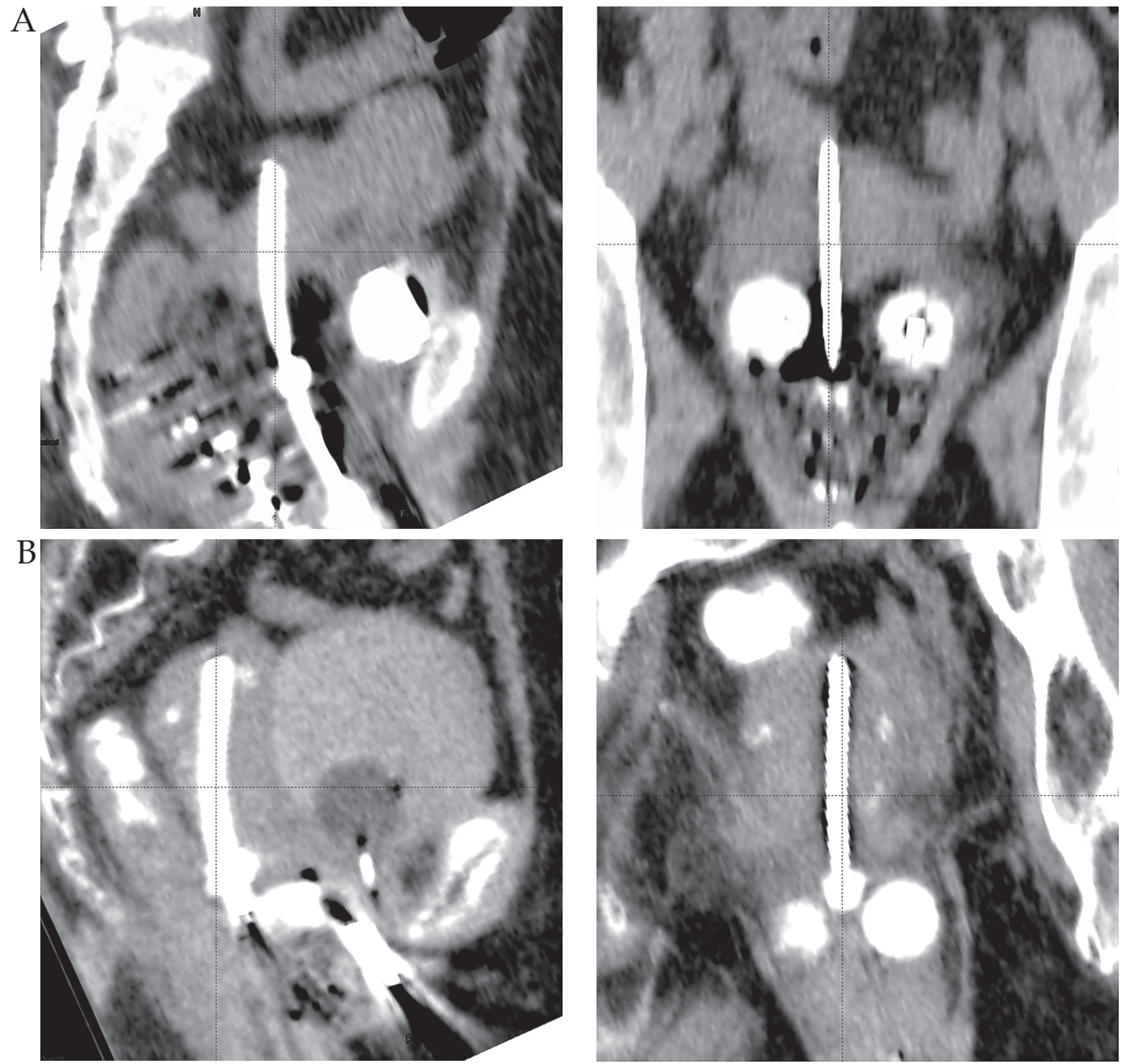

Fig. 1. Examples of uterine perforation (A) and sub-serosal insertion of intra-uterine tandem (B), in sagittal and coronal views 
Table 1. Patient characteristics $(n=82)$

\begin{tabular}{|c|c|c|c|c|}
\hline \multirow[t]{2}{*}{ Characteristics } & \multicolumn{2}{|c|}{ Optimal insertion $(n=62)$} & \multicolumn{2}{|c|}{ Sub-optimal insertion $(n=20)$} \\
\hline & $n$ & $\%$ & $n$ & $\%$ \\
\hline Brachytherapy fractions & 197 & 85.3 & 34 & 14.7 \\
\hline \multicolumn{5}{|l|}{ Age (years) } \\
\hline Range & $22-76$ & & $38-79$ & \\
\hline Median & 52 & & 55.5 & \\
\hline \multicolumn{5}{|l|}{ Stage } \\
\hline $\mathrm{IB}$ & 2 & 3.2 & 1 & 5 \\
\hline$\| \mathrm{A}$ & 3 & 4.8 & 0 & 0 \\
\hline$\| \mathrm{B}$ & 48 & 77.4 & 10 & 50 \\
\hline IIIA & 2 & 3.2 & 0 & 0 \\
\hline IIIB & 4 & 6.5 & 9 & 45 \\
\hline IVA & 1 & 1.6 & 0 & 0 \\
\hline Unknown & 2 & 3.2 & 0 & 0 \\
\hline \multicolumn{5}{|l|}{ Initial tumor size (cc) } \\
\hline Range & $1.1-510.1$ & & $4.4-189.8$ & \\
\hline Mean \pm SD & $74.7 \pm 97.1$ & & $63.4 \pm 58.3$ & \\
\hline Unknown & 19 & 30.6 & 6 & 30 \\
\hline \multicolumn{5}{|l|}{ Residual tumor size (cc) } \\
\hline Range & $0.5-90.8$ & & $0.5-20.7$ & \\
\hline Mean \pm SD & $14.4 \pm 25.8$ & & $7.1 \pm 8.7$ & \\
\hline$C R$ & 16 & 25.8 & 4 & 20 \\
\hline Unknown & 30 & 48.4 & 8 & 40 \\
\hline \multicolumn{5}{|l|}{ Site of sub-optimal insertion } \\
\hline Fundus uteri & & & 5 & 25 \\
\hline Posterior uterine wall & & & 12 & 60 \\
\hline Anterior uterine wall & & & 3 & 15 \\
\hline
\end{tabular}

$S D$ - standard deviation, $C R$ - complete response

The correlation between incidence of uterine perforation and patients' variables was assessed using logistic regression analysis. The statistical analysis of dosimetry data was performed using the Student's $t$-test and a $p$ value of $<0.05$ was considered significant.

\section{Results}

A total of 231 brachytherapy plans for 82 patients were reviewed. We identified $34(14.7 \%)$ treatment plans with sub-optimal insertion of intra-uterine tandem in 20 $(24.4 \%)$ patients: $14(6 \%)$ applications with uterine perforation and $20(8.6 \%)$ applications with sub-serosal insertion of tandem. Four patients $(4.8 \%)$ presented both uterine perforation and sub-serosal insertion. Patient clinical characteristics are summarized in Table 1.
Applicators were not removed in all sub-optimal insertions; the tip of the tandem (which was outside of the uterine canal) was not loaded and the planned radiotherapy dose was delivered. Patients were stable and managed conservatively, with no reported major complications such as excessive bleeding, sepsis or other organ injury. Although it has been noticed a correlation between patient's age (median 55.5 years) and advanced disease stage (IIB and IIIB) and the incidence of a sub-optimal insertion of intra-uterine tandem, the logistic regression analysis showed that only the stage could be a predictor for a sub-optimal implant $(p=0.005)$ but not the age $(p=0.18)$. Due to limited data regarding the initial and residual tumor volume post EBRT, its relation to the incidence of sub-optimal implants could not be analyzed. 
Table 2. Dose comparison in 14 patients between plans for sub-optimal (sub-serosal and uterine perforation) and optimal insertion of intra-uterine tandem, reported as percentage dose difference

\begin{tabular}{lccccc} 
Location & Constraints & \multicolumn{2}{c}{ Sub-serosal insertion of tandem } & \multicolumn{2}{c}{ Uterine perforation } \\
\cline { 2 - 6 } & & $\begin{array}{c}\text { Difference (\%) } \\
\text { (mean } \pm \text { SD) }\end{array}$ & $p$ & $\begin{array}{c}\text { Difference (\%) } \\
\text { (mean } \pm \text { SD) }\end{array}$ & $p$ \\
\hline CTV & $D_{90}$ & $-65.2 \pm 82.8$ & 0.025 & $-115.7 \pm 134.9$ & 0.042 \\
\cline { 2 - 6 } & $D_{100}$ & $-103.4 \pm 170.4$ & 0.078 & $-102.8 \pm 132.3$ & 0.13 \\
\hline Rectum & $D_{1 c c}$ & $-12.2 \pm 52.3$ & 0.46 & $20.2 \pm 32.7$ & 0.065 \\
\hline \multirow{nyyyyy}{*}{ Bladder } & $D_{2 c c}$ & $-12.2 \pm 48.2$ & 0.49 & $21 . \pm 30.6$ & 0.051 \\
\cline { 2 - 6 } & $D_{1 c c}$ & $-16.3 \pm 31.7$ & 0.061 & $4.1 \pm 36.7$ & 0.23 \\
\hline
\end{tabular}

CTV - clinical-target-volume, $D_{90}$ - the minimum dose to $90 \%$ of the CTV, $D_{100}$ - the minimum dose to $100 \%$ of the CTV, $D_{1 c c}-$ minimum dose to the most exposed $1 \mathrm{~cm}^{3}, D_{2 c c}-$ minimum dose to the most exposed $2 \mathrm{~cm}^{3}$

Among the 20 patients ( 34 plans) having sub-optimal implants, there were 6 patients ( 17 plans) in which all the brachytherapy plans revealed sub-optimal insertion of intra-uterine tandem, therefore they were excluded from the dosimetric analysis, as no individual reference plans were available for comparison. Dose distribution and dose-volume histograms (DVHs) analysis was performed for the remaining 14 patients (17 sub-optimal implant plans), and showed large variations when compared to the plans with optimal intra-uterine insertion. The percent differences in dose coverage of CTV (assessed by $\mathrm{D}_{90}$ ) and dose received by $1 \mathrm{cc}$ and $2 \mathrm{cc}$ of rectum and bladder are presented in Table 2.

The percent difference of $\mathrm{D}_{90}$ between sub-optimal and optimal implant plans showed a significant decrease: from $0.74 \%$ to $-319 \%$ (average: $-115.7 \% \pm 134.9 \%$ ) for uterine perforation and from $12 \%$ to $-223.8 \%$ (average: $-65.2 \% \pm 82.8 \%)$ for sub-serosal insertion of uterine tan$\operatorname{dem}(p=0.025)$, as shown in Figure 2.

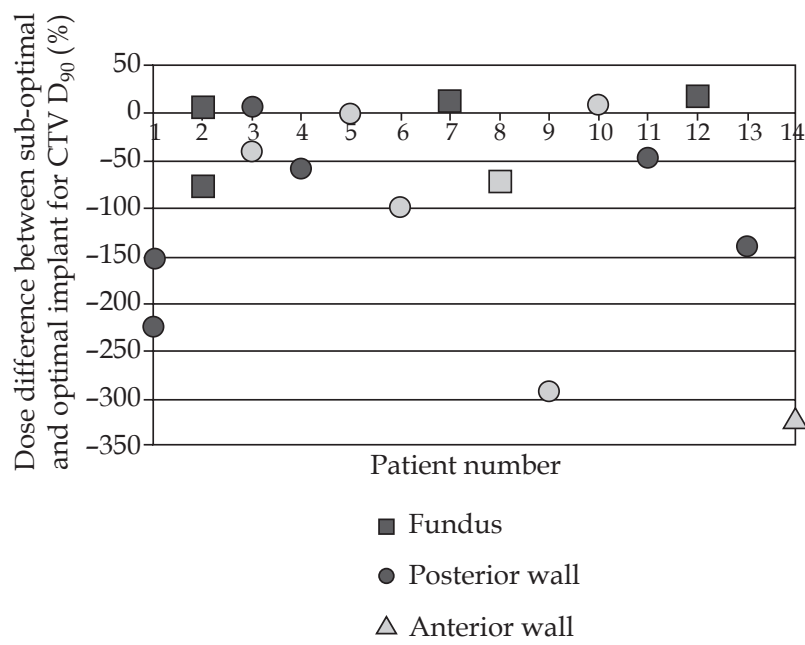

Fig. 2. Percent differences between sub-optimal implant and optimal insertion of intra-uterine tandem in the same patient for 14 patients (17 plans), regarding $\mathrm{D}_{90}$ of CTV. The black markers show the sub-serosal insertion of tandem and the gray ones the uterine perforation
The variations of the dose received by the OARs rectum and bladder were further analyzed, in order to detect a possible correlation of the dose with the position of the uterine tandem (site of perforation) relative to the uterus. The results showed that the rectum dose, assessed by $\mathrm{D}_{2 \mathrm{cc}}$ increased up to $70.3 \%$ if the sub-serosal insertion of uterine tandem occurred in the posterior uterine wall, and up to $39.7 \%$ if in the uterine fundus. The bladder dose appeared not to be so sensitive to the uterine tandem position: the maximum variations of $\mathrm{D}_{2 \mathrm{cc}}$ were $37.4 \%$ in the posterior uterine wall and $43.8 \%$ in the uterine fundus. The percent differences between sub-optimal and optimal intra-uterine tandem insertion for $\mathrm{D}_{2 \mathrm{cc}}$ of rectum and bladder are presented in Figure 3.

\section{Discussion}

Brachytherapy is an essential component of cervical cancer treatment. The technical quality of brachytherapy implant has been shown to significantly affect the clinical outcome of the treatment [9-11]. In a recent study, Viswanathan et al. showed that inappropriate placement of brachytherapy applicators led to significant risk of local recurrence (2.5 times) and lower DFS rate (2.28 times) [11].

Few published reports addressed the uterine perforation during brachytherapy, using either low-dose-rate (LDR) or HDR [7,12-14]. In the case of LDR intracavitary brachytherapy, Barnes et al. presented an incidence of CT detected uterine perforation of $13.7 \%$, although in $8.2 \%$ of insertions the physician was confident of correct tandem placement [7]. An incidence of $2 \%$ was reported by Petereit et al., using ultrasound in only $6 \%$ of cases, therefore the true occurrence of uterine perforation was probably underestimated [14]. Granai et al. employed post-operative B-mode ultrasound to evaluate final tandem position in 50 consecutive insertions in 28 patients. In 34\%, the tandem was found to be sub-optimally positioned: in $24 \%$ it penetrated the myometrium, and in $10 \%$ completely perforated the uterus [15]. In a recent study reporting one of the lowest incidence of uterine perforation in literature, Segedin et al. determined 3\% incidence of uterine perforation among 428 image-guided brachytherapy applications [16]. The authors emphasized the importance of using CT/MR imaging post insertion to accurately identify 
A

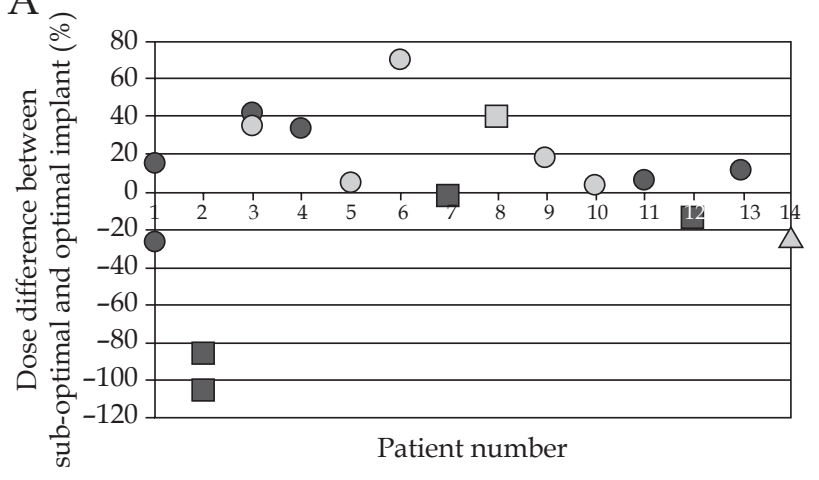

$\mathrm{B}$

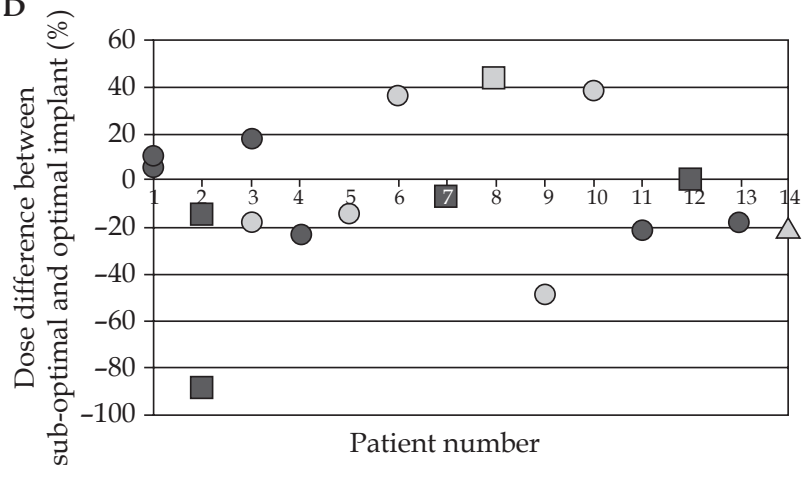

$\square$ Fundus o Posterior wall $\triangle$ Anterior wall

Fig. 3. Percent differences between sub-optimal implant and optimal insertion of intra-uterine tandem in the same patient for 14 patients (17 plans), regarding $\mathrm{D}_{2 \mathrm{cc}}$ of rectum $(\mathrm{A})$ and $\mathrm{D}_{2 \mathrm{cc}}$ of bladder $(\mathrm{B})$. The black markers show the sub-serosal insertion of tandem and the gray ones the uterine perforation

uterine perforation and used ultrasound guidance in $40 \%$ of subsequent applications after perforation.

Our study shows comparable data: $14.7 \%$ of the analyzed brachytherapy treatment plans presented sub-optimal insertion of intra-uterine tandem: $6 \%$ with uterine perforation and $8.6 \%$ with sub-serosal insertion of tandem. At our institution, uterine tandem insertion is a blind technique, performed without image guidance that would directly visualize pelvic structures, and depends on physician's clinical judgment and skills. This explains a higher incidence of perforation in our study, when compared to Segedin et al. (6\% vs. 3\%) [16]. Prior to tandem insertion, it may be difficult to predict which patients are at risk of perforation. Risk factors that have been reported include age over 60 and anatomical distortion of the cervix (due to advanced disease, cervical stenosis, radiation fibrosis, or previous cone biopsy), which can make it difficult to localize the cervical os or subsequent dilation of the endocervical canal [7,12,17-19].

Barnes et al. demonstrated that significant predictors of uterine perforation are patient age greater than or equal to $60(p=0.0019)$ and tumor size $(p=0.0016)$ [7]. Our data showed stage to be a predictor for a sub-optimal insertion $(p=0.005)$ but not the age $(p=0.18)$. Advanced disease may be associated with distortion of the cervix, difficulty to identify or localize the os, friability of cervical stroma, and uterine wall (if involved), which could increase the risk of a false track and perforation.

Nevertheless, post-insertion imaging is currently used to confirm correct uterine tandem placement, as well as to enable advanced brachytherapy planning [2024]. Given the fixed angulation of commercially available uterine tandems, perforation risk is increased in marked ante-verted or retro-verted uteri $[25,26]$. We found that $60 \%$ of the perforations occurred in the posterior uterine wall, $25 \%$ in the fundus, and $15 \%$ in the anterior wall, while Segedin et al. reported $70 \%$ in the posterior uterine wall, $15 \%$ in the fundus, and $15 \%$ in the anterior wall [16]. In addition to injuring the pelvic organs, sub-optimal in- sertion of intra-uterine tandem can lead to inadequate radiation delivery and can subsequently alter the expected clinical outcome.

Our study showed that sub-optimal uterine tandem insertion can considerably affect the quality of the brachytherapy plans. The dose coverage of CTV, assessed by $\mathrm{D}_{90}$, showed a significant decrease, with an average of $115.7 \% \pm 134.9 \%$ for uterine perforation, and $65.2 \% \pm 82.8 \%$ for sub-serosal insertion of uterine tandem $(p=0.025)$. The doses received by rectum and bladder, and evaluated by $\mathrm{D}_{2 \mathrm{cc}}$ increased with an average of $21.0 \% \pm 30.6 \%$ for rectum, and $2.1 \% \pm 36.3 \%$ for bladder, in the case of uterine perforation. While the bladder dose appeared not to be sensitive to the position of the uterine tandem relative to the uterine cavity, the rectum dose markedly increased when the sub-optimal insertion of tandem occurred in the posterior uterine wall.

Several reports investigated the effect of applicator displacement on brachytherapy plan dosimetry, and emphasized the importance of image guidance and comprehensive quality control in the practice of brachytherapy [27-30]. However, published data on the effect of mal-placement of brachytherapy applicators on plan quality are scarce, hence a comparison with our data was not possible. Furthermore, the clinical significance of carrying out brachytherapy with a sub-optimally inserted intra-uterine tandem is not yet determined.

Pelvic magnetic resonance (MR) imaging after EBRT and before brachytherapy is a useful modality to assess the residual tumor and the orientation of uterus. For patients predicted to be at risk of perforation, ultrasound-guided insertion of applicators should be considered to minimize patient morbidity [21]. Intracavitary brachytherapy procedures are gradually becoming image-guided. Various authors have used X-ray fluoroscopy, ultrasonography, CT scan, MRI, and laparoscopy for guiding brachytherapy procedures in gynecological malignancies. Methods such as CT and MR scans have practical limitations and are rarely used. Transrectal ultra- 
sound (TRUS) can provide real time imagery of pelvic structures and movement of applicators, and thus potentially help in avoiding perforation and injury to OAR. It has many advantages in terms of easy availability, simple, and cost effective equipment, free from ionizing radiation [31]. Even though ultrasound-guidance is known to reduce the risk of uterine perforation, it is not yet used routinely by many clinicians [32].

The use of traditional X-ray simulations may make the planner unaware of the true position of the central tandem, which if loaded in the intra-abdominal location could risk the small intestine to be exposed to serious radiation dosages [33]. Post-insertion imaging techniques such as ultrasound, CT or MR, have been shown to detect uterine perforation in up to $66 \%$ of cases when clinical assessment suggested proper tandem placement, and have been recommended by several authors [19-22,33,34]. Some authors have even suggested additional pelvic imaging following tandem insertion for all patients [7,35]. Image-guided brachytherapy has a double advantage in that it not only allows the detection of inadequate placement of the applicators but also gives the opportunity of dose optimization during the planning process.

An obvious limitation of the present study is the small number of patients presenting uterine perforation. Statistical analysis of such a small sample size could not be valid if generalized at patient population level, and future studies with large sample sizes are recommended.

\section{Conclusions}

We report relatively low incidence rates of uterine perforation and sub-serosal insertion of uterine tandem in CT-guided intracavitary HDR brachytherapy for cervix carcinoma, without image guidance during applicators insertion. The effects on CTV coverage and rectal dose were considerably detrimental. Based on our data, we advise image-guided tandem insertion, at least for challenging cases.

\section{Disclosure}

Authors report no conflict of interest.

\section{References}

1. NCCN Guidelines Version 1.2015. Cervical Cancer, 2015.

2. National Cancer Institute. Cervical Cancer Treatment, 2014.

3. Haie-Meder C, Morice P, Castiglione M. Cervical cancer: ESMO Clinical Practice Guidelines for diagnosis, treatment and follow-up. Ann Oncol 2010; 21: 37-40.

4. Eifel PJ, Thomas WW, Smith TL. The relationship between brachytherapy dose and outcome in patients with bulky endocervical tumors treated with radiation alone. Int J Radiat Oncol Biol Phys 1994; 28: 113-118.

5. Lanciano RM, Won M, Coia L. Pretreatment and treatment factors associated with improved outcome in squamous cell carcinoma of the uterine cervix: a final report of the 1973 and 1978 patterns of care studies. Int J Radiat Oncol Biol Phys 1991; 20: 667-676.
6. Perez CA, Kao MS. Radiation therapy alone or combined with surgery in the treatment of barrel-shaped carcinoma of the uterine cervix (stages IB, IIA, IIB). Int J Radiat Oncol Biol Phys 1985; 11: 1903-1909.

7. Barnes EA, Thomas G, Ackerman I et al. Prospective comparison of clinical and computed tomography assessment in detecting uterine perforation with intracavitary brachytherapy for carcinoma of the cervix. Int J Gynecol Cancer 2007; 17: 821-826.

8. Irvin W, Rice L, Taylor P et al. Uterine perforation at the time of brachytherapy for carcinoma of the cervix. Gynecol Oncol 2003; 90: 113-122.

9. Corn BW, Hanlon AL, Pajak TF et al. Technically accurate intracavitary insertions improve pelvic control and survival among patients with locally advanced carcinoma of the uterine cervix. Gynecol Oncol 1994; 53: 294-300.

10. Eifel PJ, Morris M, Wharton JT et al. The influence of tumor size and growth habit on outcome of patients with FIGO stage IB squamous cell carcinoma of the uterine cervix. Int J Radiat Oncol Biol Phys 1994; 29: 9-16.

11. Viswanathan AN, Moughan J, Small W et al. The quality of cervical cancer brachytherapy implantation and the impact on local recurrence and disease-free survival in radiation therapy oncology group prospective trials 0116 and 0128 . Int J Gynecol Cancer 2012; 22: 123-131.

12. Kim RY, Levy DS, Brascho DJ et al. Uterine perforation during intracavitary application. Prognostic significance in carcinoma of the cervix. Radiology 1983; 147: 249-251.

13. Matsuyama T, Tsukamoto N, Matsukuma $\mathrm{K}$ et al. Uterine perforation at the time of brachytherapy for the carcinoma of the uterine cervix. Gynecol Oncol 1986; 23: 205-211.

14. Petereit DG, Sarkaria JN, Chappell RJ. Perioperative morbidity and mortality of high-dose-rate gynecologic brachytherapy. Int J Radiat Oncol Biol Phys 1998; 42: 1025-1031.

15. Granai CO, Allee P, Doherty F et al. Intraoperative real-time ultrasonography during intrauterine tandem placement. Obstet Gynecol 1986; 67: 112-114.

16. Segedin B, Gugic J, Petric P. Uterine perforation - 5-year experience in 3-D image guided gynaecological brachytherapy at Institute of Oncology Ljubljana. Radiol Oncol 2013; 47: 154160 .

17. Jhingran A, Eifel PJ. Perioperative and postoperative complications of intracavitary radiation for FIGO stage I-III carcinoma of the cervix. Int J Radiat Oncol Biol Phys 2000; 46: 1177-1183.

18. Corn BW, Shaktman BD, Lanciano RM et al. Intra- and perioperative complications associated with tandem and colpostat application for cervix cancer. Gynecol Oncol 1997; 64: 224-229.

19. Granai CO, Doherty F, Allee P et al. Ultrasound for diagnosing and preventing malplacement of intrauterine tandems. Obstet Gynecol 1990; 75: 110-113.

20. Beddy P, Rangarajan RD, Sala E. Role of MRI in intracavitary brachytherapy for cervical cancer: what the radiologist needs to know. AJR 2011; 196: 341-347.

21. Watkins JM, Kearney PM, Opfermann KJ et al. Ultrasound-guided tandem placement for low-dose-rate brachytherapy in advanced cervical cancer minimizes risk of intraoperative uterine perforation. Ultrasound Obstet Gynecol 2011; 37: 241-244

22. Addley HC, Vargas HA, Moyle PL et al. Pelvic imaging following chemotherapy and radiation therapy for gynecologic malignancies. Radiographics 2010; 30: 1843-1856.

23. Nag S, Erickson B, Thomadsen B et al. The American Brachytherapy Society recommendations for high-dose-rate brachytherapy for carcinoma of the cervix. Int J Radiat Oncol Biol Phys 2000; 48: 201-211. 
24. Sahinler I, Cepni I, Oksuz DC et al. Tandem application with transvaginal ultrasound guidance. Int J Radiat Oncol Biol Phys 2004; 59: 190-196.

25. Prabhakar GS, Revannasiddaiah S, Susheela SP et al. Uterine perforation during intracavitary brachytherapy for carcinoma of the cervix. BMJ Case Reports 2012; doi:10.1136/bcr2012-007830.

26. Mayr NA, Montebello JF, Sorosky JI et al. Brachytherapy management of the retroverted uterus using ultrasound-guided implant applicator placement. Brachytherapy 2005; 4: 24-29.

27. Garipagaoglu M, Tuncel N, Dalmaz MG et al. Changes in applicator positions and dose distribution between high dose rate brachytherapy fractions in cervix carcinoma patients receiving definitive radiotherapy. Br J Radiol 2006; 79: 504-509.

28. Yoshida K, Ueda M, Takenaka T et al. Daily CT measurement of needle applicator displacement during multifractionated high-dose-rate interstitial brachytherapy for postoperative recurrent uterine cancer. J Radiat Res 2012; 53: 295-300.

29. Koushik K, Bilimagga R, Rao N et al. Positional variation of applicators during low dose rate intracavitary brachytherapy for cervical cancer: a prospective study. J Contemp Brachytherapy 2010; 2: 93-97.

30. Tanderup K, Hellebust TP, Lang S et al. Consequences of random and systematic reconstruction uncertainties in 3D image based brachytherapy in cervical cancer. Radiother Oncol 2008; 89: 156-163.

31. Sharma DN, Rath GK, Thulkar S et al. Use of transrectal ultrasound for high dose rate interstitial brachytherapy for patients of carcinoma of uterine cervix. J Gynecol Oncol 2010; 21: 12-17.

32. Davidson MT, Yuen J, D'Souza DP et al. Optimization of high-dose-rate cervix brachytherapy applicator placement: the benefits of intraoperative ultrasound guidance. Brachytherapy 2008; 7: 248-253.

33. Makin WP, Hunter RD. CT scanning in intracavitary therapy: unexpected findings in "straightforward" insertions. Radiother Oncol 1988; 13: 253-255.

34. Rotmensch J, Waggoner SE, Quiet C. Ultrasound guidance for placement of difficult intracavitary implants. Gynecol Oncol 1994; 54: 159-162.

35. Small W Jr, Strauss HB, Hwang CS et al. Should uterine tandem applicators ever be placed without ultrasound guidance? No: a brief report and review of the literature. Int J Gynecol Cancer 2011; 21: 941-944. 